\title{
Aspectos teóricos a considerar para la generación de un modelo no formal y participativo de educación ambiental orientado a la protección y conservación de los humedales Bañó y Los Negros, Colombia
}

\author{
Theoretical aspects to consider for the generation of a \\ non formal participative model of environmental education \\ orientated towards the protection and conservation \\ of the wetlands Bañó and Los Negros, Colombia
}

Jorge Villadiego-Lorduy'

Dennis Huffman-Schwocho

Adolfo Cortecero-Bossio ${ }^{3}$

Antonio Ramos-Ruiz ${ }^{4}$

Fecha de recepción: I de abril del 2013

Fecha de aprobación: 7 de agosto del 2013

Villadiego, j; Huffman, D; Cortecero, A; Ramos, A.

Aspectos teóricos a considerar para la generación de un modelo no formal y participativo de educación ambiental orientado a la protección y conservación de los humedales Bañó y Los Negros, ColombiaTecnología en Marcha.Vol. 26, № 4. Pág 92-99

I Geógrafo. Estudiante del programa de Doctorado en Ciencias Naturales para el Desarrollo, Instituto Tecnológico de Costa Rica. Correo electrónico: villalordl5@yahoo.es. Tel.: 3135058 180. Servicio Nacional de Aprendizaje (SENA). Departamento de Vichada, Cumaribo, Colombia.

2 Doctor en Pedagogía. Profesor titular del programa de Doctorado en Ciencias Naturales para el Desarrollo. Tel.: 2475-53 I0. Correo electrónico: d_huffman@yahoo.com. Instituto Tecnológico de Costa Rica. Costa Rica.

3 Ing. Químico. Msc en Gestión Ambiental. Tel.: 3 I 45346748. Correo electrónico: acobo7l@yahoo.com. Fundación Kontiqui. Departamento de Bolívar, Cartagena de Indias, Colombia.

4 Geógrafo. Especialista en Gestión Ambiental. Tel.: 3215264I38. Correo electrónico: antjrr_2l@yahoo.es. Fundación Salud Mental para Todos. Departamento de Córdoba, Montería, Colombia. 


\section{Palabras clave}

Perfil ambiental; metodología; base pedagógica.

\section{Resumen}

Los problemas ambientales existentes en los humedales de Bañó y los Negros son el resultado de las presiones de tipo antrópico a las que han sido sometidos, ante la ausencia de una cultural ambiental en los habitantes de las comunidades vecinas. El resultado ha sido el deterioro de sus ecosistemas. Como respuesta, se pretende aplicar uno de los cuatro instrumentos de la gestión ambiental, la educación ambiental, entendida como un proceso sistémico que, partiendo del conocimiento reflexivo y crítico de la realidad biofísica, social, política, económica y cultural, permita al individuo comprender las relaciones de interdependencia con su entorno. A partir de esto, se busca debatir aspectos teóricos que se puedan incluir al momento de proponer un modelo de educación ambiental no formal y participativo, dando respuesta a una pregunta base y teniendo en cuenta aportes teóricos como la educación andragógica, el aprendizaje constructivista y significativo, los enfoques sistémico, interdisciplinario, comunitario e interpretativo, la inclusión de valores y actitudes ambientales, la metodología acción-participación, al igual que la encuesta participativa. De la misma forma, la población objeto de estudio la integran personas de 20 a 55 años de edad que que ya poseen una serie de conocimientos sobre su entorno. Es prioritario el desarrollo de un diagnóstico territorial que permita obtener una valoración real del área de estudio y así poder identificar los factores que están influyendo en tal deterioro ambiental, como también determinar la base pedagógica y los elementos a considerar para la generación del modelo pedagógico ambiental.

\section{Key words}

Environmental profile; methodology; pedagogic basis.

\section{Abstract}

The existing environmental problems in the wetlands of Bañó and Los Negros are the result of the anthropic pressures to which they have been submitted; in the absence of cultural environmental ones, their inhabitants have spoilt present day ecosystems. In response to this, one of the four instruments of environmental management is applied, environmental education; understood as a systemic process that, based on the reflexive and critical knowledge of biophysical, social, political, economic and cultural realities, allows the individual to understand relationships of interdependence with his or her environment. Considering the previously mentioned above, a debate of theoretical aspects that could be included at the moment of proposing a non formal participative model of environmental education is intended, in order to respond to a series of basic questions that bring theoretical contributions to mind such as andralogical education, constructivist and significant learning, systemic interdisciplinary approaches, interpretive communities, the incorporation of values and environmental attitudes, and action-participation methodologies such as a participative survey. In the same way, the population, object of study, is integrated by adult persons whose ages range from 20 to 55 years and who possess a series of previous knowledge of their environment. As a conclusion, the development of a territorial diagnosis that allows for a true valuation of the area of study is considered a priority which will make it possible to identify the factors that are influencing environmental deterioration, and therefore also determine the pedagogic basis and central elements needed for the generation of a pedagogic environmental model. 


\section{Introducción}

La problemática ambiental se ha acelerado y agudizado en las últimas décadas. Hoy día la globalización económica impone nuevas pautas para la producción y consumo de recursos. Por lo tanto, la educación es una vía útil y necesaria para potenciar al máximo la formación y capacitación ambiental en distintos ámbitos de la sociedad, desde los tomadores de decisiones hasta los ciudadanos, cuya actuación diaria incide en forma directa sobre el medio (Novo, 1998:19). Los orígenes de la educación ambiental se encuentran en los años 70, aunque el término ya aparece en documentos de la Organización de las Naciones Unidas para la Ciencia, la Educación y la Cultura (UNESCO) de 1965, sin embargo, no es sino hasta 1972 en Estocolmo, durante la conferencia de la Organización de las Naciones Unidas sobre el Medio Humano, que se reconoce oficialmente la existencia de este concepto y de su importancia para cambiar el modelo de desarrollo.

Desde entonces, se han realizado diferentes actividades, conformando el llamado debate ambiental. Entre ellas se destacan el Coloquio Internacional sobre la Educación Relativa al Medio Ambiente (Belgrado, 1975), en el cual se definieron los objetivos y líneas de acción de la educación ambiental; igualmente importante fue la Conferencia Intergubernamental sobre Educación Ambiental, organizada por la UNESCO y el Programa de las Naciones Unidas para el Medio Ambiente (PNUMA) en Tbilisi, ex URSS, en 1977, que planteó la incorporación de la educación ambiental en los sistemas educativos de los estados participantes, concretando sus funciones y principios, así como el diseño y elaboración de material y la participación activa de los ciudadanos en todos los niveles sociales.

La educación ambiental (EA) se ha convertido en un poderoso instrumento de la gestión ambiental y debe entenderse como un proceso dinámico y participativo, que busca despertar en la población una conciencia que le permita identificarse con la problemática tanto a nivel general como específico. Guier (2002) define la EA como "un proceso inherente a todo espacio educativo, conducente a la formación de personas despiertas a su realidad y conscientes de que pertenecen a un sistema ecológico global, regido por una serie de leyes y principios que deben conocerse y sobre todo respetarse para garantizar la vida misma". Commoner (citado por
Guier, 2002) señala cinco leyes básicas que se deben comprender en este tipo de educación:

I. La interdependencia, donde todo está relacionado con lo demás.

2. Todo debe ir a alguna parte, por lo tanto, la materia se transforma, no se crea ni se destruye.

3. La naturaleza sabe lo que hace, los sistemas naturales tiene sus roles bien definidos.

4. No existe la comida en balde, en los sistemas naturales existen cadenas y redes tróficas perfectamente equilibradas.

5. Los recursos naturales son limitados, el planeta y la energía proveniente del sol tienen límites.

Por otra parte, la educación ambiental se divide en tres categorías: formal, no formal e informal. La primera está inmersa en el sistema educativo tradicional y organizada en un currículo de carácter transversal. La segunda está fundamentada en la transmisión de conocimientos, aptitudes y valores ambientales fuera del sistema educativo tradicional y que conllevan a que las personas beneficiarias desarrollen aptitudes positivas hacia el ambiente, su fin no es alcanzar certificaciones o títulos. La última y no menos importante se da de forma espontánea y no planificada, no responde a estructuras pedagógicas.

En el mismo sentido, Bohm (citado por Guier, 2002) establece unos principios que caracterizan la EA y su implementación, basados en el enfoque sistémico e interdisciplinar, resolución de problemas, metodología participativa, reconocimiento de la biodiversidad y de la sociodiversidad, solidaridad, pensamiento constructivo y enfoque interpretativo. Covas (2004) apunta a varios de esos principios y enfatiza la pertinencia de que la educación ambiental se trabaje bajo tres enfoques: comunitario, sistémico e interdisciplinario.

Desde el enfoque comunitario, se hace necesario involucrar a las personas en el análisis de los problemas ambientales de sus territorios, generar pensamientos desde lo local hacia lo global con el fin de proponer alternativas de solución. El enfoque sistémico da herramientas para analizar la realidad de forma holística; aquellas personas que son capacitadas en una comprensión sistémica de la complejidad ambiental y que participan en el ámbito comunitario son capaces de diseñar futuros posibles y deseables (Trellez, 2006). Asimismo, la interdisciplinariedad 
permite hacer aportaciones desde las diferentes áreas del saber de forma articulada.

Cabe resaltar que los problemas ambientales son el resultado de un modelo social, económico y cultural cuya rectificación es tarea de todos. Los problemas ambientales de los países industrializados son distintos de aquellos de los países en desarrollo. Los problemas asociados con el ambiente se caracterizan por su complejidad. Son básicamente abiertos y solo determinados aspectos se pueden abordar de forma cercana (Artieda, 1999).

En el marco de la educación ambiental no formal, Guier (2002) señala que ésta es responsabilidad de organizaciones gubernamentales y no gubernamentales, además prioriza las capacitaciones y la producción de materiales didácticos. El grupo meta se define según los objetivos del programa o modelo a implementar y existe una relación estrecha en que también se contemplan los sistemas a distancia.

Novo (2005) señala una serie de características implícitas a este tipo de educación y que se relacionan con los enfoques propuestos por Covas y Bohm anteriormente, como son el contexto, el desarrollo de procesos interdisciplinares, la conciencia participativa, la flexibilización del rol profesor/estudiante, la creación de redes, los múltiples recursos y vías de aprendizaje y la estimulación de relaciones de educación/trabajo. Igualmente, el mismo autor enfatiza en los principios y valores que fundamentan la educación ambiental no formal. Para Novo (2005), es importante comprender el concepto de interdependencia tanto por parte de los educadores como de los educandos, es decir, la interrelación de las esferas socioeconómicas y biofísicas como un todo y las repercusiones que traería si se llegase a alterar el medio, entender el planeta como una unidad. Asimismo, la necesidad de generar pensamientos globales, pero con actuaciones locales por parte de los individuos y la construcción de valores que permitan orientar el actuar de las personas de manera favorable al ambiente, teniendo en cuenta los conocimientos previos que ellos poseen.

No menos importante como principio aplicado a la enseñanza de la educación ambiental es el enfoque interdisciplinario, basado en la articulación y cooperación de los distintos saberes con el fin de encontrar soluciones a los problemas ambientales. No obstante, en cuanto a la participación ciudadana se refiere y como un elemento clave de la educación ambiental no formal, existe la necesidad de fomentar el sentido de pertenencia como agente activo de los individuos en las comunidades, con el fin de aportar de modo puntual a las problemáticas ambientales locales. De este modo, cada persona involucrada en procesos de capacitación ambiental siente que desde su lugar de acción puede contribuir a revertir esa realidad conflictiva (Iris, 2007).

\section{Consideraciones acerca de la generación}

un modelo no formal de educación

ambiental participativo para los

humedales Bañó y Los Negros

Los humedales de Bañó y Los Negros se encuentran en el Bajo Sinú, exactamente entre los corregimientos de Cotocá Arriba, Castilleral y Palo de Agua, todos pertenecientes al área rural del Municipio de Lorica, departamento de Córdoba, Colombia. La primera está localizada a 09008'09" N y 075 $50^{\circ}$ ' I "

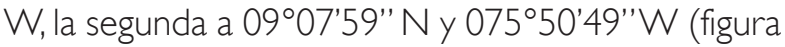
I).

Actualmente, las presiones a la que se ven sometidos ambos espejos por parte de las comunidades vecinas son constantes. Sus pobladores en muchos casos no poseen una cultura ambiental que favorezca la interacción con el medio, es decir, carecen de valores ambientales, acarreando con ello problemas en los entornos biofísico y social. Hoy día se encuentran individuos no sensibilizados con el ambiente, apáticos para participar en la solución de los problemas existentes. Se señala la poca cooperación entre las comunidades y la falta de reflexión sobre los impactos de tipo negativo que éstas le producen al medio. Ambos espejos de agua tienen problemas de sedimentación, taponamiento de los caños que alimentan los humedales, eutrofización y colmatación, caza y comercialización de especies y tala indiscriminada, entre otros. Cabe resaltar que las poblaciones vecinas de los citados humedales no han sido sometidas a procesos o iniciativas ambientales de educación en sí, resultando en un desarrollo escaso de experiencias previas en educación ambiental no formal. Además se desconoce la importancia que tienen estas ciénagas para la supervivencia de las comunidades, lo cual ha contribuido de forma directa a la depredación de estos ecosistemas.

Para proponer un modelo pedagógico ambiental que responda a una problemática contextualizada 
y particular, es necesario preguntarse ¿cuál debe ser el modelo no formal de educación ambiental participativo que se genere para la protección y conservación de los humedales Bañó y los Negros? Con el fin de responder al interrogante planteado, se prioriza el desarrollo del perfil ambiental del territorio, el cual debe contribuir a determinar el estado real en que se encuentran los humedales y las áreas aledañas, caracterizando los problemas ambientales y las esferas social y económica (en este aparte es clave la recopilación de información primaria y secundaria aplicando instrumentos como cuestionarios, matrices, fichas de contenido, diario de campo y técnicas como la observación directa e indirecta, análisis, síntesis y estadística descriptiva, entre otros).

Por otra parte, una vez identificadas las debilidades, fortalezas, amenazas y oportunidades del área de estudio, se busca establecer los factores que están influyendo en las conductas ambientalmente negativas de sus habitantes, a partir de un análisis situacional soportado en el perfil ambiental y en la percepción y cultura ambiental que poseen los habitantes de los humedales hacia estos. Hasta el momento se ha determinado una serie de problemas ambientales (taponamiento de caños, deterioro de ecosistemas, tráfico de especies y una serie de necesidades básicas insatisfechas) y poca cultura ambiental, que podrían relacionarse como factores de presión hacia los recursos naturales existentes. En tal sentido, y como un referente para el levantamiento de la línea base territorial se señala a Sánchez et al. (2009), quienes aplicaron un enfoque social para el diagnóstico de la cuenca del rio San Diego (Cuba), sus resultados abarcaron la identificación de dificultades sociales así como la creación de talleres comunitarios y de una propuesta de educación ambiental, entre otros aspectos. Es prioritario, desde la educación ambiental no formal, enseñarle a las comunidades a valorar los territorios aledaños a los humedales de Bañó y Los Negros.

Asímismo, la población objeto de estudio oscila entre los 20 y 55 años de edad, tratándose de población adulta. Por lo tanto, los individuos que integran este universo poblacional poseen ciertos conocimientos previos que se pueden tener en cuenta para originar procesos de aprendizaje significativos. Al respecto,

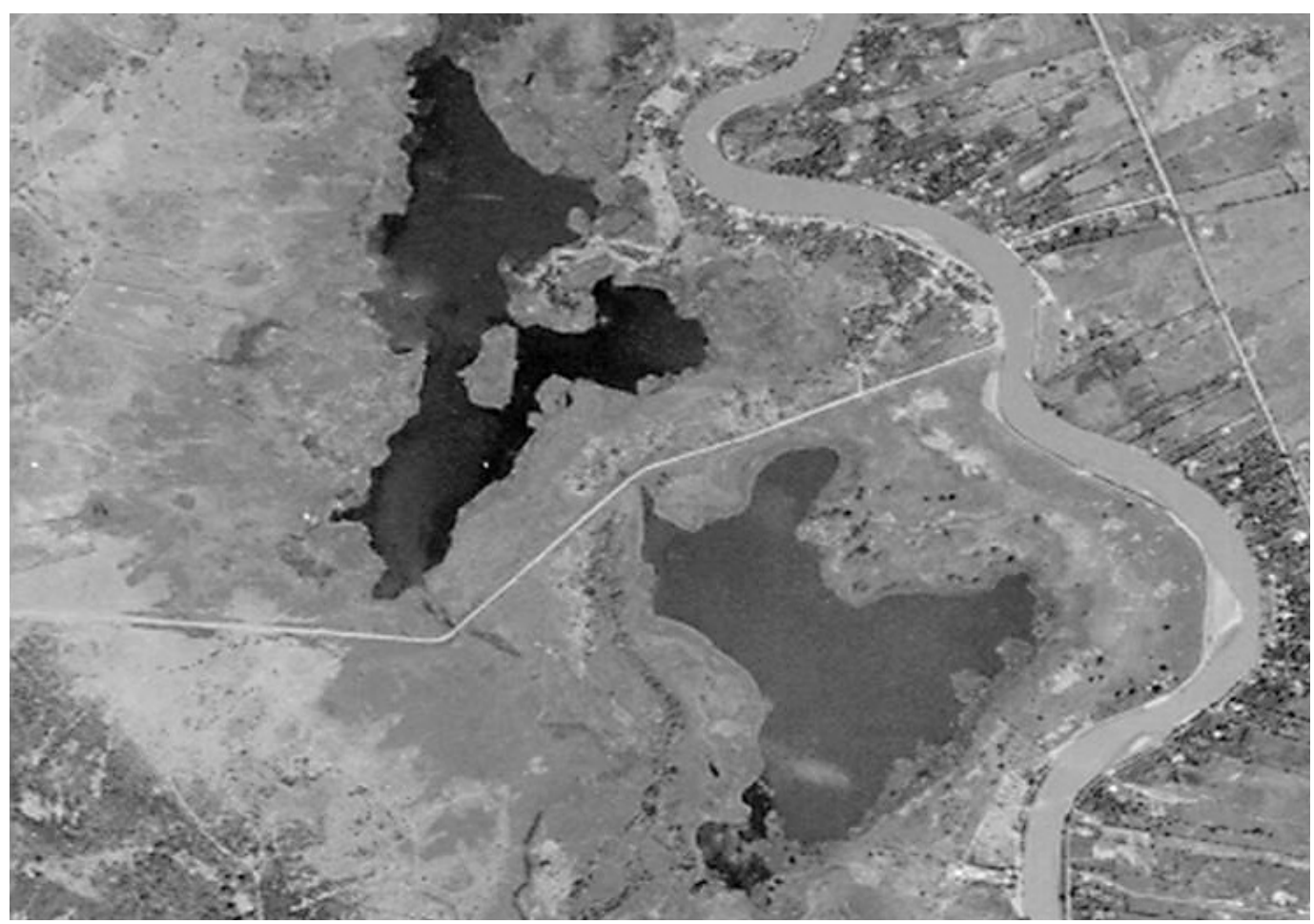

Figura I. Fotografía aérea de las ciénagas de Bañó y Los Negros, margen izquierdo del río Sinú, en el corregimiento de Cotoca, Municipio de Lorica. Ciénaga de Bañó (parte inferior de la foto) y Ciénaga Los Negros (parte superior). IGAC 1994. 
Moreno (2002) indica que la sistematización de la educación en adultos se concreta en la andragogía, teniendo en cuenta que el ser humano durante su vida recibe influencias socioambientales y que este tipo de educación adopta diversas formas según los lugares, el tiempo, el nivel de aprendizaje, etc. Igualmente, para Cruz (2003) la función social de la educación, en cualquiera de sus niveles pero especialmente en la atención a los adultos, se hace objeto de una acción formativa estable y continuada en el tiempo. Es a través de un constante flujo de informaciones entre el individuo y el grupo social que se va desarrollando un cuerpo de actitudes, creencias, valores, comportamientos, así como capacidades y habilidades, que también serán reflejo de su intervención y participación en los desempeños del grupo social cotidiano al que pertenece.

Un aspecto relevante que debe considerarse e incluirse en un proceso de educación ambiental no formal se centra en los valores. Elzo (2004) propone una serie de ellos entre los que destacan la solidaridad, la espiritualidad, la tolerancia activa, la racionalidad y la competencia personal. En el mismo sentido, Orellana (1996) considera dos metodologías útiles para la educación ambiental: la encuesta participativa y la acción-participación. Tanto la encuesta participativa como la acciónparticipación tratan básicamente de la inclusión de los individuos en el análisis crítico de una situación y de sus problemas y en la elaboración de acciones educativas destinadas a resolverlos.

Para el caso de las comunidades aledañas a los humedales de Bañó y Los Negros, es prioritario que comprendan los alcances del modelo y que se apropien de él, que no lo perciban como un proyecto que se desarrolla en las comunidades sino como un pilar para su desarrollo. Cid (2005) manifiesta que entre los resultados obtenidos en el estudio "Educación, comunicación e interpretación en los espacios naturales protegidos: El caso de los humedales RAMSAR en España", está que solo un 15\% de los casos analizados manifiesta la voluntad de entender los programas de educación ambiental como instrumento para la gestión de los humedales, un 75\% de los programas para la conservación de los humedales se centra solamente en la divulgación de valores ambientales y tan solo un 10\% señala como objetivos de los programas la participación en la conservación de los espejos de agua.
Se percibe entonces con base en los datos proporcionados por Cid que el modelo no formal de educación ambiental debe dirigirse no solo hacia la divulgación de valores ambientales sino a involucrar a la comunidad en el proceso de conservación de los humedales Bañó y Los Negros.

En cuanto a las metodologías aplicadas en los programas de EA, básicamente la visita de campo es la más utilizada. Sería interesante tener en cuenta en el modelo algunas de las teorías que fundamentan la educación ambiental para el desarrollo sostenible propuestas por Gutiérrez y Pozo (2006), (citando Max-Neef, 1998), acerca de la teoría sustentable basada en un modelo de desarrollo a escala humana capaz de dar respuesta a las necesidades básicas de subsistencia, protección, afecto, entendimiento, participación, ocio, creación, identidad y libertad, según un prototipo de economía respetuoso de los recursos naturales.

La problemática ambiental de los espejos de agua de Bañó y Los Negros es el resultado de una mal interacción de sus habitantes con el medio, un desequilibrio entre las esferas social, económica y físicobiótica que ha desencadenado alteraciones en el territorio.

De lo anterior, y teniendo en cuenta los aportes teóricos expuestos, se pueden considerar como posibles aspectos integradores del modelo varios enfoques, que abarcan desde el sistémico y el comunitario hasta el interdisciplinar, el interpretativo y el de desarrollo sostenible. Igualmente se podrían referenciar como metodologías de trabajo la encuesta participativa y la acción-participación, además de aspectos de modelos pedagógicos de tipo constructivista, de aprendizaje significativo y educación andragógica, que contribuyan al fomento de valores y aptitudes favorables al entorno (Ver figura 2).

\section{Conclusiones}

Como resultado del análisis teórico-metodológico de distintos antecedentes de estudios especializados relacionados con el diseño e implementación de programas de educación ambiental no formal y participativa para adultos, consideramos importante que:

- El modelo pedagógico ambiental que se genere debe responder a las particularidades del territorio, para lo cual es prioritario tener en cuenta 


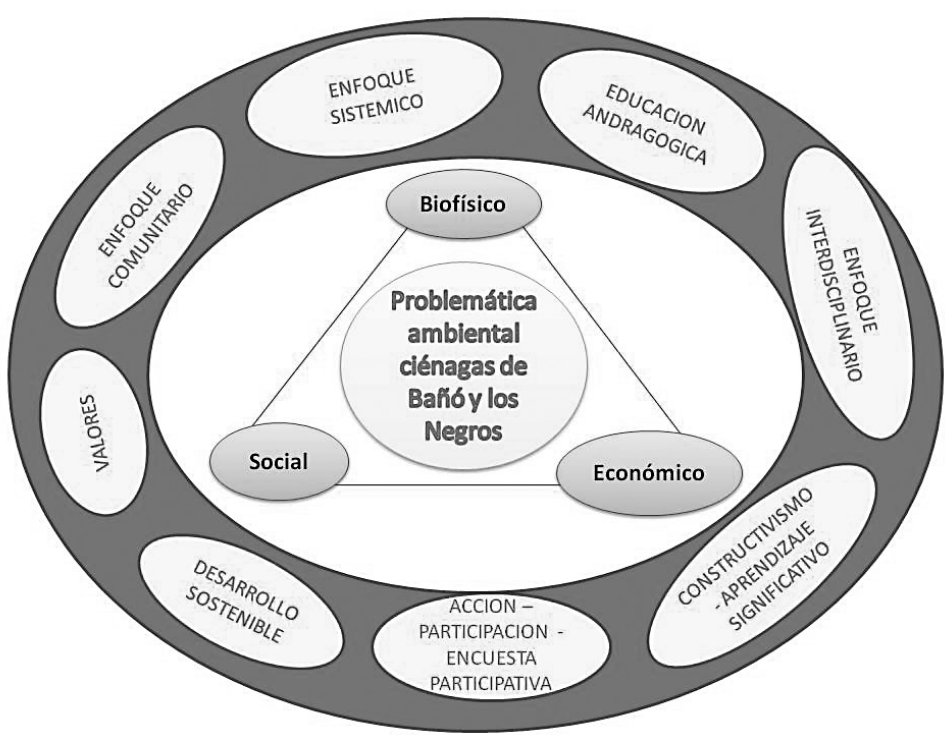

Figura 2. Aspectos teóricos y metodológicos a considerar en un modelo no formal de educación ambiental para la protección y conservación de los humedales Bañó y Los Negros. Fuente: Elaboración propia.

el contexto del área de estudio, la población a la que va dirigido y la realización de un diagnostico del territorio a profundidad.

- En términos de la base pedagógica acorde para el modelo, esta debe basarse en elementos de educación andragógica y aprendizaje significativo, teniendo en cuenta los resultados o experiencias obtenidas por expertos que han desarrollado este tipo de estudios.

- Igualmente importante es el enfoque sobre el cual se trabajará el estudio, señalándose como fundamentales los aspectos sistémicos, comunitarios e interdisciplinarios del modelo pedagógico que se utilice.

- En términos de metodologías, la acción-participación resulta muy acorde para el modelo, pues la comunidad se convierte en coinvestigadora y altamente participativa en el proceso de investigación.

\section{Recomendaciones}

Como observaciones para retomar en la realización del presente estudio pedagógico, de carácter principalmente cualitativo, se debe estar consciente de ciertos aspectos importantes de analizar en el diseño metodológico:
- En este tipo de investigaciones es necesario lograr que los miembros de la comunidad se involucren como coinvestigadores de las problemáticas ambientales de sus territorios, así como generadores de las posibles soluciones.

- Sería interesante que cada uno de los habitantes del territorio donde existe la problemática ambiental se apropien del modelo no formal de educación ambiental, haciéndose participantes activos de su implementación y mejoramiento.

- Es muy importante que las autoridades ambientales brinden apoyo a los habitantes de las comunidades aledañas a los humedales y coadyuven a la articulación de cada uno de los actores sociales, económicos e institucionales que se encuentren inmersos en los problemas ambientales.

- En términos de la educación no formal, se deben tener en cuenta los conocimientos previos que tengan los individuos sobre su medio y generar un aprendizaje significativo que ayude a la comprensión de su entorno y las relaciones que se dan en él.

Consideramos que los elementos pedagógicos señalados en estas conclusiones y recomendaciones son centrales para lograr cabalmente la coherencia en el diseño de un modelo de educación ambiental no 
formal y participativo, y así tener un mayor impacto positivo sobre la resolución de la problemática ambiental de los humedales de Bañó y Los Negros, en el Departamento de Córdoba, Colombia.

\section{Bibliografía}

Artieda, G. (abril, 1999). Educación ambiental: cuestiones básicas. (En línea). Revista Lurralde 22: 279-298. Consultado el 3 de sept. de 2012. Disponible en http://www.ingeba.org/ lurralde/lurranet/lur22/goran22/22ogran.htm

Cid, O. (octubre, 2005). Educación, comunicación e interpretación en los espacios naturales protegidos: El caso de los humedales RAMSAR en España. En: Nuevas Tendencias en Investigaciones en Educación Ambiental (en línea). Organismo Autónomo Parques Nacionales. Consultado el 19 ago. 2012. Disponible en http://www.oei.es/decada/ portadas/nuevas_tendencias.pdf.

Covas, O. (noviembre, 2004). La educación ambiental a partir de tres enfoques: Comunitario, sistémico e interdisciplinario (en línea). Revista Iberoamericana de Educación 34: I-7. Consultado el 15 de ago. de 2012. Disponible en http:// www.rieoei.org/deloslectores/794Covas.PDF.

Cruz, M. (febrero, 2003). El ámbito de la educación no formal como espacio de formación civil: la participación ciudadana. (En línea). Revista Ágora 4: I-10. Consultado 14 de Ene de 2013. Disponible en http://www.uhu.es/agora/version0l/ digital/numeros/04/04articulos/monografico/pdf_4/04.PDF

Elzo, J. (Junio, 2004). La educación del futuro y los valores. (En línea). Fundación Jaume Bofill, UOC. Ponencia debates de educación. Págs. I-14. Consultado el 30 de ago de 2012. Disponible en http://www.uoc.edu/dt/esp/elzo0704.pdf.
Guier, E., Rodríguez, M. \& Zúñiga, M. (2002). Educación Ambiental en Costa Rica: Tendencias Evolutivas, Perspectivas y Desafíos. San José: Editorial UNED. 99 p.

Gutiérrez, J. \& Pozo,T. (enero, 2006). Modelos teóricos contemporáneos y marcos de fundamentación de la educación ambiental para el desarrollo sostenible. (En línea). Revista Iberoamericana de Educación 41:21-68. Consultado el I5 de ago. de 2012. Disponible en http://www.rieoei.org/rie4 I a0 I. pdf.

Iris, A. (octubre, 2007). Talleres comunitarios de educación ambiental para la introducción del pensamiento ambiental a nivel local (En línea). Revista Delos Vol. I, No. 0: I - I3. Consultado el 15 de feb. de 2013. Disponible en http://www.eumed.net/ $\mathrm{rev} / \mathrm{del}$ os/00/sia.pdf.

Max-Neef, M. (1998). Desarrollo a escala humana, Barcelona, Icaria.

Moreno, F. \& Quintero, M. (enero, 2002). La educación andragógica: Una estrategia frente a los problemas ambientales. (En línea). Revista Actualidad Contable FACES No. 5: 7-22. Consultado el 15 de nov. de 2012. Disponible en http://www.redalyc.org/articulo.oa?id=25700506.

Novo, M. (septiembre, 2005). Educación ambiental y educación no formal: dos realidades que se realimentan. (En línea). Revista de Educación 338: 145-165. Consultado el 14 de ene. de 2013. Disponible en http://www.revistaeducacion. mec.es/re338_09.htm

Novo, María (1998) "La educación ambiental: bases éticas, conceptuales y metodológicas" Ediciones UNESCO, editorial Universitas, S.A. Madrid, España.

Sánchez, A., Reyes, L. \& Cruz, J. (diciembre, 2009). Enfoque social en el diagnóstico ambiental de la cuenca del río San Diego. (En línea). Revista sociedad de la información I 8: I - 12. Consultado el 14 de ene. de 2013. Disponible en http:// www.sociedadelainformacion.com/ / 8/Enfoque.pdf 